\title{
Multi-objective unit commitment problem using Cuckoo search Lagrangian method
}

\author{
*K. Chandrasekaran and Sishaj P. Simon \\ Department of Electrical and Electronics Engineering, National Institute of Technology, Tiruchirappalli-620015, INDIA \\ Email: chansekaran23@gmail.com, sishajpsimon@nitt.edu \\ *Corresponding author: Phone: +91-0431-2513298 Fax: +91-0431-2500133
}

\begin{abstract}
Restructuring of power system changed the mechanism of reliability management in solving the unit commitment problem (UCP). In general, operating reserve capacity is predetermined either by dispatch rules or predefined by reliability index. This paper considers that the operating reserve capacity in a power system is bendable, which is based on the cost-reliability issues. In UCP, these two competing objectives such as fuel cost and reliability level of the system were optimized simultaneously, as a multi-objective unit commitment problem (MOUCP), using the proposed fuzzy integrated binary real coded cuckoo search Lagrangian (BCSL) algorithm. The ON/OFF status of the thermal power units is obtained by binary coded cuckoo search algorithm (CSA), where as the economic dispatch is obtained by Lagrangian multiplier method. The fuzzy set theory is used to pick up the best compromise solution. The proposed methodology is tested and validated for both the single and multi-objective UCP. The effectiveness of the proposed technique is demonstrated on IEEE RTS 24 bus system by comparing its performance with other methods reported in the literature.
\end{abstract}

Keywords: binary coded cuckoo search algorithm, fuzzy set theory, reliability index, reserve scheduling, unit commitment problem.

DOI: http://dx.doi.org/10.4314/ijest.v4i2.7

\section{Introduction}

Unit commitment is a nonlinear mixed integer optimization problem to schedule the operation of the thermal power units at minimum operating cost, satisfying the demand and other equality and inequality constraints. UCP is to find the commitment schedule (ON/OFF status of generators) and thereby the power output level of each committed thermal power unit. This scheduling has to be done daily for the time interval planned (hourly) for dispatch, in order to minimize the total fuel cost. Minimization of total cost which includes the fuel cost, start up, and shut down cost is carried out by satisfying load demand, spinning reserve, physical and operational constraints, of the individual thermal power unit.

Power system operators have to keep a certain amount of generation capacity as spinning reserve. Thereby, it ensures that the power system is able to withstand the sudden outage of some thermal power units/ transmission lines or an unforeseen increase in load without having to resort to load shedding. The traditional criterion for setting the minimum amount of spinning reserve is that it should be at least equal to the capacity of the largest thermal power unit, or to a specific percentage of the hourly system load. While deterministic criterion is easy to implement, since they do not match the stochastic nature of the problem and do not take into consideration of the intrinsic reliability of each scheduled generator. They lead to inconsistent decision and variable operating risk levels.

During the last 40 years, numerous techniques and methods have been developed to incorporate probabilistic reserve criteria in the formulation of the reserve constrained UCP (Dillon et. al., 1978; Gooi et al., 1999; Chattopadhyay. and Baldick, 2002; Bouffard and Galiana, 2004; Simopoulos, 2006; Wu, 2008). Later, methods provide promising result in the evaluation of spinning reserve by including various system risks. The choice of appropriate values for setting probabilistic reserve criterion depends on 
the global preference information of the system operator or decision-maker. Hence, an analysis is carried out in this article to demonstrate the effect of reserve criteria on unit commitment solution and the results are presented in section 7.2. From the result it is clear, that setting of reserve criterion by the decision makers will affect the solution of UCP and system reliability. Hence this method is more suitable only when the entire global preference information of the decision-maker is available. Therefore, maximizing the reliability level has to be handled as another objective function simultaneously, in addition to the cost. Hence the system becomes highly non-smooth in nature.

Many solution strategies are available to solve the highly non-smooth problem. A literary review of UCP and the solution techniques are given in (Sheble and Fahd, 1994; Padhy, 2004). Techniques like priority list method (Burns and Gibson, 1975), dynamic programming (Synder, 1987), mixed integer programming (Muchkstadt and Wilson, 1968), branch and bound (Chen and Wang, 1993; Cohen and Yoshimura 1983) and Lagrangian Relaxation (Zhuang and Galiana, 1988) are the widely used conventional techniques. The priority list method is simple and fast. However, it produces sub-optimal solution with higher operation cost. Dynamic programming method has dimensionality problem. That is with increase in problem size, the solution time increases rapidly with the number of thermal power units to be committed. Though LR method provides a fast solution, it suffers from numerical convergence and the solution quality due to the dual nature of the algorithm, is poor. In branch-and-bound method, the computational time increases enormously for a large-scale power system. So, artificial intelligence techniques like, neural networks (Ouyang and Shahidehpour, 1992), expert systems (Wang. and Shahidehpour, 1992), genetic algorithm (GA) (Dasgupta and McGregor, 1994; Kazarlis et al., 1996; Pavez-Lazo and Soto-Cartes, 2011), simulated annealing (SA) (Mantaway. et. al., 1998a), evolutionary programming (EP) (Juste et al., 1999), tabu search (Mantaway et al., 1998b), fuzzy logic (Saneifard, 1997), particle swarm optimization (PSO) (Ting et al., 2006; Saber et al. 2007; Yuan et al., 2009;), ant colony optimization (ACO) (Simon et al. 2006) and hybrid algorithm (Lin et. al., 2011; Patra et al. 2009;) are used. These are population based search techniques and can search for the global or near global optimal solution for any large-scale system incorporating all system constraints, with ease. In expert system, interaction with the plant operators are required making it inconvenient for a realistic system. Though GA and PSO has an immense potential for applications in the field of power systems and it has been successfully applied to solve various problems in electric power systems such as economic dispatch, unit commitment, reactive power control, hydrothermal scheduling, and distribution system planning etc.

GA searches multiple solutions simultaneously in contrast to conventional optimal algorithms. Therefore, the possibility of finding global optimal solution is increased. The main advantage of GA is that it finds near optimal solution in relatively short time compared with other random search methods. Despite the aforementioned success, GA is only capable of identifying the high performance region at an affordable time and displays inherent difficulties in performing local search for numerical applications. Also the characteristics of GA exhibit premature convergence and settles to near-global optima. In general, the PSO contains parallel search techniques. However, similar to the GA, the main adversity of the PSO is premature convergence, which might occur when the particle and group best solutions are trapped into local minimums during the search process. Localization occurs because particles have the tendency to fly to local, or near local, optimums, therefore, particles will concentrate to a small region and the global exploration ability will be weakened. Also many techniques are developed to solve UCP, no technique has been accepted as the best so far. In this context, an attempt is made to solve single and multi-objective UCP using a newly developed bio inspired algorithm (Gandomi and Alavi, 2012) such as cuckoo search algorithm (CSA) to demonstrate the significant important of proposed method on solving large scale optimization problems.

\section{Proposed Work}

The main interest of this paper is to show the efficiency of the cuckoo search algorithm (Yang and Deb, 2009; Yang and Deb, 2010; Rajabioun, 2011) in solving UCP and to propose a novel MOUCP to eliminate the role of the decision maker (system operator) in setting the spinning reserve (either by deterministically or probabilistically) in UCP. In MOUCP problem, similar to other evolutionary methods, cuckoo search algorithm starts with an initial fixed number of cuckoo nests. At the end of every generation of CSA, the size of cuckoo nests increases when compared to the initial number of nests. Hence the fuzzy set theory (Cai. et. al., 2010; Wang and Singh, 2007; Vinothkumar and Selvan, 2011) is used to select the best compromise solution (which is equal to the initial number of nests) that would be alive for the coming generations. Inclusion of fuzzy set theory will thrown away or abandon the solution (nest) which is a far away from the best solution.

Hence in this article, the binary coded CSA is used to obtain the unit commitment scheduling for the 24 hour and Lagrangian multiplier method (Hemamalini and Simon, 2012) is used to obtain the economic dispatch for the 24 hour.

\section{Problem formulation}

Modeling of two objective functions fuel cost and reliability function is presented below.

\subsection{Objective function 1: Fuel Cost Function, $F_{c}$}

The fuel cost minimization problem is formulated as: Minimize 


$$
F_{c}=\sum_{k=1}^{H} \sum_{i=1}^{N} F_{c}(P(i, k)) * I(i, k)+\sum_{k=1}^{H} S T(k)=\sum_{k=1}^{H} \sum_{i=1}^{N}\left(\left(a(i)+b(i) \cdot P(i, k)+c(i) \cdot P(i, k)^{2}\right)\right)+\sum_{k=1}^{H} S T(k)
$$

\subsection{Objective function 2: Reliability Function, LOLP}

In UCP the system reliability level is dependent on the allocation of spinning reserve. Hence, to minimize $L O L P$, enough spinning reserve has to be scheduled for each hour. Reliability level of the power system at each hour is calculated using (2)

$$
\operatorname{LOLP}(k)=\sum_{j=L C} p(j), k \in[1, H]
$$

\subsection{Problem Constraints}

3.3.1. Power balance constraints

$$
\sum_{i=1}^{N}(P(i, k) * I(i, k))=\operatorname{Load}(k), k \in[1, H]
$$

3.3.2. Spinning reserve constraints:

$$
\sum_{i=1}^{N}\left(P(i, \max )^{*} I(i, k)\right) \geq \operatorname{Load}(k), k \in[1, H]
$$

3.3.3. Capacity limits of thermal power unit

$$
P(i, \min ) \leq P(i, k) \leq P(i, \max )
$$

3.3.4. Thermal power unit minimum ON/OFF durations

$$
\begin{aligned}
& {\left[X^{\text {on }}(i, k)-T^{o n}(i)\right]^{*}[I(i, k-1)-I(i, k)] \geq 0} \\
& {\left[X^{\text {off }}(i, k-1)-T^{\text {off }}(i)\right]^{*}[I(i, k-1)-I(i, k)] \geq 0}
\end{aligned}
$$

3.3.5. Thermal power unit ramp constraints

$$
\begin{aligned}
& P(i, k)-P(i, k-1) \leq R U(i) \\
& P(i, k-1)-P(i, k) \leq R D(i)
\end{aligned}
$$

\section{Implementation of multi-objective function}

Real time optimization problems involve simultaneous optimization of different conflicting objectives. Hence a multi-objective optimization problem consists of multiple objectives to be optimized simultaneously with the various equality and inequality constraints. This can be generally formulated as

$$
\begin{aligned}
\text { Min } f_{i}(x), i=1,2, \ldots, N O & \\
\text { subject to } & :\left\{\begin{array}{l}
g_{j}(x)=0, \quad j=1,2, \ldots, M \\
h_{k}(x) \leq 0, \quad k=1,2, \ldots, K
\end{array}\right.
\end{aligned}
$$

where $f_{i}$ is the $i^{\text {th }}$ objective function, $x$ is a decision vector that represents a solution, $N O$ is the number of objective functions, $M$ and $K$ are the number of equality and inequality constraints, respectively. Multi-objective optimization problems with such conflicting objectives, give raise to a set of optimal solution, rather than a single optimal solution, since, no solution can be considered to be better than other solutions, without adequate information. These set of optimal solutions are called as Paretooptimal solutions (Zitzler, E. and Thiele L., 1999). For practical applications, however, we need to select one solution, which will satisfy the different goals to some extent. Such a solution is called best compromise solution. One of the challenging factors of the tradeoff decision is the imprecise nature of the decision maker's judgment.

In the proposed methodology, fuzzy set theory has been integrated with BCSL method to extract a Pareto optimal solution as the best compromise solution. In the fuzzy set theory, it is required to formulate a fuzzy membership function for each objective function, i.e. the corresponding membership function value should indicate the associated degree of satisfaction for that objective. 
Therefore fuzzy membership function should be framed in such a manner that it aids BCSL in maximizing the fitness function. The fuzzy memberships function for each objective functions are defined below.

\subsection{Fuzzy Membership Function for $F_{c}$}

The design of the membership function implies that for any solution, if the objective function, $F_{c}$ in the fuzzy domain is more than $F_{c \operatorname{cmax}}$, then the associated fuzzy membership function value is assigned to be zero. Such solutions (corresponding nest positions) do not maximize the fitness function and won't take part in the optimal solution set. On the other hand, if the objective function is less than $F_{c m i n}$, then the associated fuzzy membership function value is assigned to be unity. Such solutions do participate and maximize the fitness function. If the objective function in the fuzzy domain is between $F_{c \min }$ and $F_{c \max }$, then the associated fuzzy membership function value is computed using equation (12), and such solutions will participate in the optimization process depending on the fitness value.

$$
\mu^{c}= \begin{cases}1, & \text { for } F_{c}<F_{c \min } ; \\ \frac{\left(F_{c}-F_{c \min }\right)}{\left(F_{c \max }-F_{c \min }\right)}, & \text { for } F_{c \min } \leq F_{c} \leq F_{c \max } ; \\ 0, & \text { for } F_{c}>F_{c \max }\end{cases}
$$

where $F_{c}$ is the degree of the objective function $F_{c}$ in the fuzzy domain.

\subsection{Fuzzy Membership Function for LOLP}

The design of the membership function implies that, for an objective function of LOLP in the fuzzy domain which is less than $F_{r m i n}$, the system cost is increased proportionally. Hence the fuzzy membership function value is assigned to be zero. Such solutions (corresponding firefly positions) do not participate in the optimal solution set. On the other hand, when the objective function of $L O L P$ in fuzzy domain is greater than $F_{\text {rmax }}$, it will affect the system reliability and hence won't take part in the optimal solution set. If the value of the objective function in the fuzzy domain is between $F_{r \min }$ and $F_{r \max }$, then the associated fuzzy membership function value is computed using equation (13) and such solutions will participate in the optimization process depending on the fitness value.

$$
\mu^{r}= \begin{cases}0, & \text { for } F_{r} \leq F_{r \text { min }} ; \\ \frac{\left(F_{\text {ravg }}-F_{r}\right)}{\left(F_{\text {ravg }}-F_{r \text { min }}\right)}, & \text { for } F_{r \text { min }}<F_{r}<F_{\text {ravg }} ; \\ 1 & \text { for } F_{r}=F_{\text {ravg }} ; \\ \frac{\left(F_{r \max }-F_{r}\right)}{\left(F_{r \text { max }}-F_{\text {ravg }}\right)}, & \text { for } F_{\text {ravg }}<F_{r}<F_{r \text { max }} ; \\ 0, & \text { for } F_{r}>F_{r \max } ;\end{cases}
$$

where $F_{r}$ is the degree of the objective function $L O L P$ in the fuzzy domain.

\section{Overview of Cuckoo Search Algorithm}

In this section, the breeding behavior of cuckoos and the characteristics of cuckoos in reaching best habitat societies are discussed. Cuckoo search algorithm (CSA) is one of the most recently defined algorithms (Yang, X.S. and Deb S., 2009; Yang, X.S. and Deb S., 2010) by Xin-She Yang and Suash Deb in 2009. It has been developed by simulating the intelligent breeding behavior of cuckoos. It is a population-based search procedure that is used as an optimization tool, in solving complex, nonlinear and non-convex optimization problems.

The cuckoo bird searches the most suitable nest to lay eggs (solution) in order to maximize their eggs survival rate. Each cuckoo lays one egg at a time. The eggs (high quality of eggs i.e. near to optimal value) which are more similar to the host bird's eggs have the opportunity to develop (next generations) and become a mature cuckoo. Alien eggs (away from optimal value) are detected by host birds with a probability $P a \varepsilon[0,1]$ and these eggs are thrown away or the nest is abandoned, and a completely new nest is built, in a new location. The mature cuckoo form societies and each society have its habitat region to live in. The best habitat from all of the societies will be the destination for the cuckoos in other societies. Then they immigrate towards this best habitat.

A randomly distributed initial population of host nest is generated and then the population of solutions is subjected to repeated cycles of the search process of the cuckoo birds. The cuckoo randomly chooses the nest position to lay egg using equations (14$15)$.

$$
V_{p q}^{g e n+1}=V_{p q}^{g e n}+s_{p q} \times L^{\prime} \operatorname{evy}(\lambda) \times \alpha
$$




$$
L^{\prime} \operatorname{evy}(\lambda)=\left|\frac{\Gamma(1+\lambda) \times \sin \left(\frac{\pi \times \lambda}{2}\right)}{\Gamma\left(\frac{(1+\lambda)}{2}\right) \times \lambda \times s^{\frac{(\lambda-1)}{2}}}\right|^{\frac{1}{\lambda}}
$$

where $\lambda$ is a constant $(1<\lambda \leq 3)$ and $\alpha$ is a random number generated between $[-1,1]$. Also $s>0$ is the step size which should be related to the scales of the problem of interests. If $s$ is too large, then the new solution generated will be too far away from the old solution (or even jump out of the bounds). Then, such a move is unlikely to be accepted. If it is too small, the change is too small to be significant, and consequently such search is not efficient. So a proper step size is important to maintain the search as efficiently as possible. Hence the step size is calculated using equation (16).

$$
s_{p q}=V_{p q}^{g e n}-V_{f q}^{g e n}
$$

where $p, f \in\{1,2, \ldots, m\}$ and $q \in\{1,2 \ldots D\}$ are randomly chosen indexes. Although $f$ is determined randomly, it has to be different from $p$. $D$ is the number of parameters to be optimized and $m$ is the total population of nest position.

Using equation (14) the cuckoo chooses the nest, and the egg which is laid by a cuckoo, is evaluated. The host birds identified the alien egg (solution away from the optimal value) and chose the high quality of egg with the probability value associated with that quality of egg, using equation (17).

$$
\text { Pro } p=\left(0.9 * \mathrm{Fit}_{p} / \max (\text { Fit })\right)+0.1
$$

where Fit $_{p}$ is the fitness value of the solution $p$ which is proportional to the quality of egg in the nest position $p$. The egg is discovered by the host bird by comparing randomly (i.e. probability $\mathrm{Pa} \in[0,1]$ ) with Pro $_{p}$. If the host bird discovers the alien egg, the host bird can either throw the egg away or abandon the nest, and build a completely new nest using equation (18). Otherwise, the egg grows up and is alive for the next generation.

$$
V_{p}=V_{p \min }+\operatorname{rand}(0,1) \times\left(V_{p \max }-V_{p \min }\right)
$$

where $V_{p \min }$ and $V_{p \max }$ are the minimum and maximum limits of the parameter to be optimized.

\section{Implementation of Binary CSA}

In MOUCP, binary numbers 1 and 0 are used to indicate the thermal power unit status ON/OFF. The CSA used in (Yang, X.S. and Deb S., 2009; Yang, X.S. and Deb S., 2010) is essentially a real-coded algorithm, thus, some modifications are needed to deal with binary-coded optimization problem. In the proposed binary CSA, the relevant variables are interpreted in terms of changes of probabilities. Now cuckoo randomly chooses the nest position, to lay egg, using equation (14). In (14), the step size is calculated using equation (16). When $\lambda$ is varied between the limits, the corresponding variation in (15) is between 0 and 1 . From these values, it is observed that the equation (14) is varied between -1 and 2 . The threshold level can be fixed within $(0,1)$. Generally to accomplish this, sigmoid (Wang and Singh., 2009) function is used as in equation (19).

$$
f\left(V_{p q}\right)=\frac{1}{1+\exp \left(-V_{p q}\right)}
$$

However, to improve the performance of binary CSA, another function called tanh is used as given in (20).

$$
f\left(V_{p q}\right)=\tanh \left(\left|V_{p q}\right|\right)=\frac{\exp \left(2 * \mid V_{p q}\right)-1}{\exp \left(2 *\left|V_{p q}\right|\right)+1}
$$

Both the functions, scales the $V_{p q}$ value within $[0,1]$ as shown in Fig. 1 . A random number is generated between 0 and 1 , to decide the thermal power unit status as 0 or 1 . If $\mathrm{f}\left(V_{p q}\right)$ is greater than rand $(0,1)$ then, the unit status is 1 otherwise 0 . The chances of bit flipping are found to be more in the case of the tanh function than in the sigmoid function. From the number of trails, it is observed that the performance of the tanh function on reaching quality solution is faster when compared to the sigmoid function. 


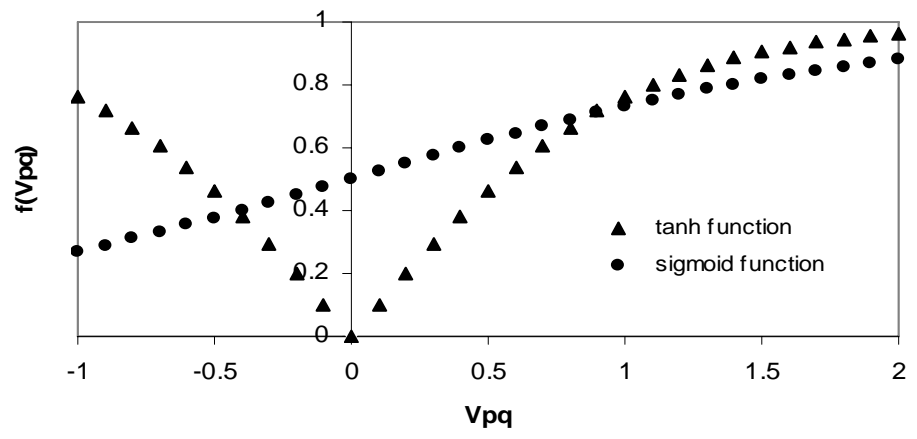

Fig. 1 Variation of $V_{p q}$ values and the function of $V_{p q}$ values.

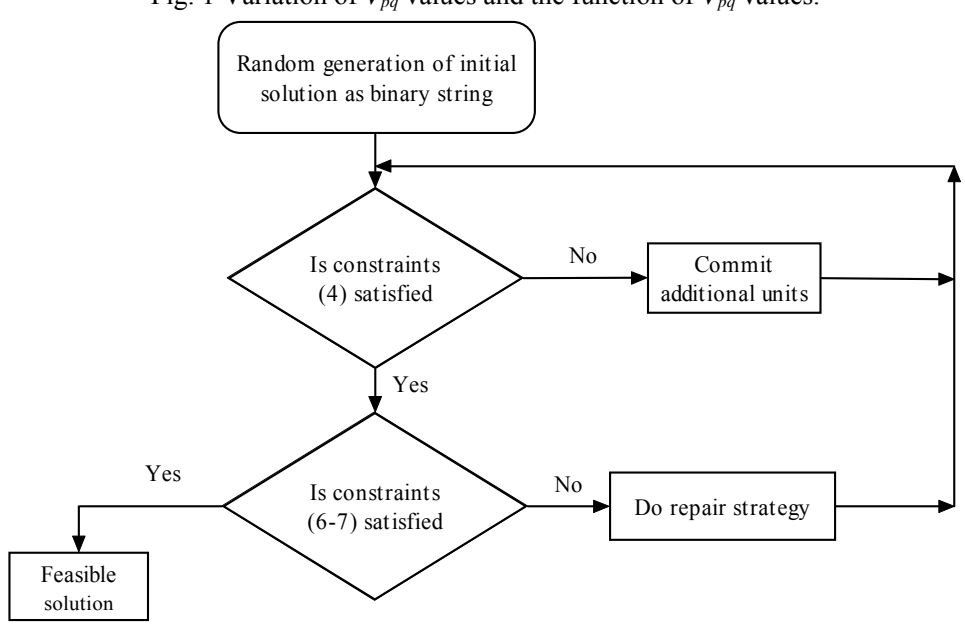

Fig. 2. Flowchart on the repair strategy.

\subsection{Repair Strategy for Constraint Management in Binary Coded CSA}

Whenever the commitment status for each time interval is generated randomly or by the modification of nest position, violation of minimum up/ down time constraints (6-7) and spinning reserve constraint(4) have to be checked as follows:

Step 1: If the spinning reserve (4) is met, then go to step 3. Otherwise, go to the next step.

Step 2: The less expensive thermal power units which are in the OFF state are identified and turned ON. Then go to step 1.

Step 3: If the spinning reserve constraint is satisfied, then the minimum up and down time constraints (6-7) are checked for each thermal power unit. If there is any violation in the minimum up or down time constraint then a repair scheme is performed to overcome the violation. For instance, let us assume that the $T^{\text {on }}$ and $T^{\text {off }}$ for a hypothetical thermal power unit is 4 and 5 . For a scheduling interval of 12 hours, if the actual OFF time for that thermal power unit is 3 hours $\left(5^{\text {th }}-7^{\text {th }}\right.$ hour $)$, then it violates the $T^{\text {off }}$ constraint. In this case, the thermal power unit status before $5^{\text {th }}$ hour or after $7^{\text {th }}$ hour should be made OFF. By doing this change, if it violates the $T^{\text {on }}$ constraint, then the status of the thermal power units are made ON corresponding to the thermal power unit status.

Step 4: The repair scheme in step 3 may affect the spinning reserve constraint of the system. If the reliability level is met, then accept the feasible solution. Otherwise go to step 1.

A minimum number of trials should be set for the repair mechanism. These steps are carried out for the entire hourly load. This is represented in Fig. 2.

\subsection{Binary Coded CSA for UCP}

Step: 1 Initialize the CSA parameters such as the nest size, lambda value and maximum generation number.

Step: 2 Initial Generation of Binary String Population (Binary Coded CSA)

Randomly generate a population of $M$ initial solutions represented by a binary string. Initialize randomly an initial population $M=\left[X_{1} ; X_{2} ; X_{3} ; \ldots \ldots ; X_{m}\right]$ of $m$ solutions or host nest positions in the multi dimensional solution space where $m$ represents the population of host nest. Each solution of $X$ is represented by the D-dimensional vector. Here $D$ is equal to $N^{*} H$. A population of $M$ initial solution with $D$ dimensional vector is shown in Fig. 3 .

Step: 3 Generation $=1$

Step: 4 Modification of host nest position 
The host nest produces a modification on the current position using (14). The modified position is then checked for constraints in (4) and (6-7).

\section{Step: 5 Repair and Evaluate EDP}

The randomly generated commitment status for each time interval is checked for spinning reserve constraint (4) and minimum up/down time (6-7) constraint violation. In case of any violation, the binary strings are repaired as in section 6.1. Then, economic load dispatch is done for the feasible positions and constraints (3) and (5) are satisfied. Here, ED problem is solved using Lagrangian multiplier method (Hemamalini and Simon, 2012).

\section{Step: 6 Fuzzy Fitness Evaluations}

A fuzzification mechanism and fitness sharing are introduced in this CSA to pick out the best compromise solution. For each non dominated solution, the normalized membership fuzzy fitness function (Fit) is calculated using equation (21)

$$
\text { Fit }_{p}=\frac{\left(\mu_{p, k}^{c}+\mu_{p, k}^{r}\right)}{\sum_{p=1 t o m}\left(\mu^{c}{ }_{p, k}+\mu^{r}{ }_{p, k}\right)}
$$

where $m$ is the total number of solutions (nest position). The best compromise solution is one having high quality of egg compared to the other nest's.

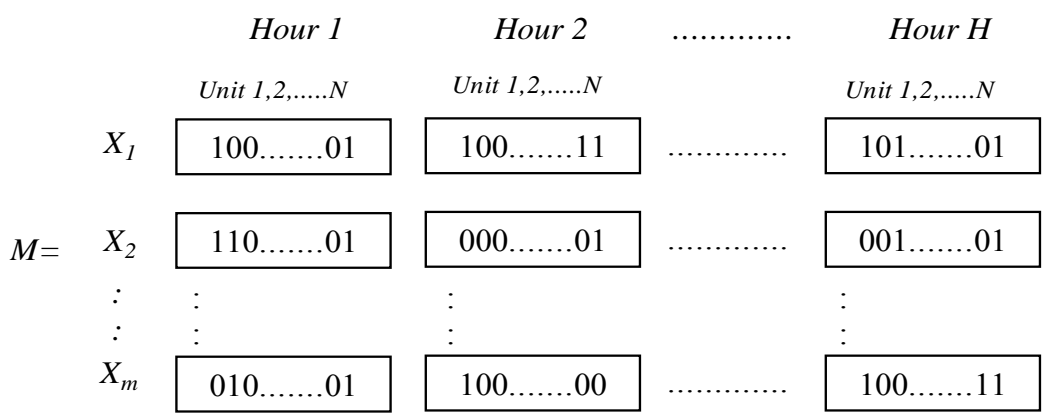

Fig. 3 Initialization of binary string

\section{Step: 7 Identification of Alien Egg}

Each nest position host bird discovered the alien egg with probability Pro $_{p}$ using equation (17) related to its fitness value. New nest position is generated using (18) and added into the existing nest population.

\section{Step: 8 Fitness Evaluation for newly added nest position}

For the new nest position, constraints (4) and (6-7) are checked for spinning reserve and minimum up time and down time constraints violations. If there is any violation in the constraints then they undergo the repair mechanism as mentioned in step 2.

Then EDP is performed and the fitness is evaluated

\section{Step: 9 Abandon sources exploited by the cuckoos}

Arrange fitness function in descending order and pick up the first $m$ number of nest position and abandoned the remaining nest position.

Step: 10 Memorize the best solution achieved so far. Increment the generation count.

Step: 11 Stop the process if the termination criterion is satisfied. Termination criteria used in this work is the specified maximum number of generation. Otherwise, go to step 4. The best fitness and the corresponding position of the host nest position is retained in the memory at the end of the termination criteria is selected as the optimum commitment schedule of thermal power units involved in the power generation process for the scheduling time interval. The step-by-step procedure for the proposed method is given as a flowchart in Fig.4. 


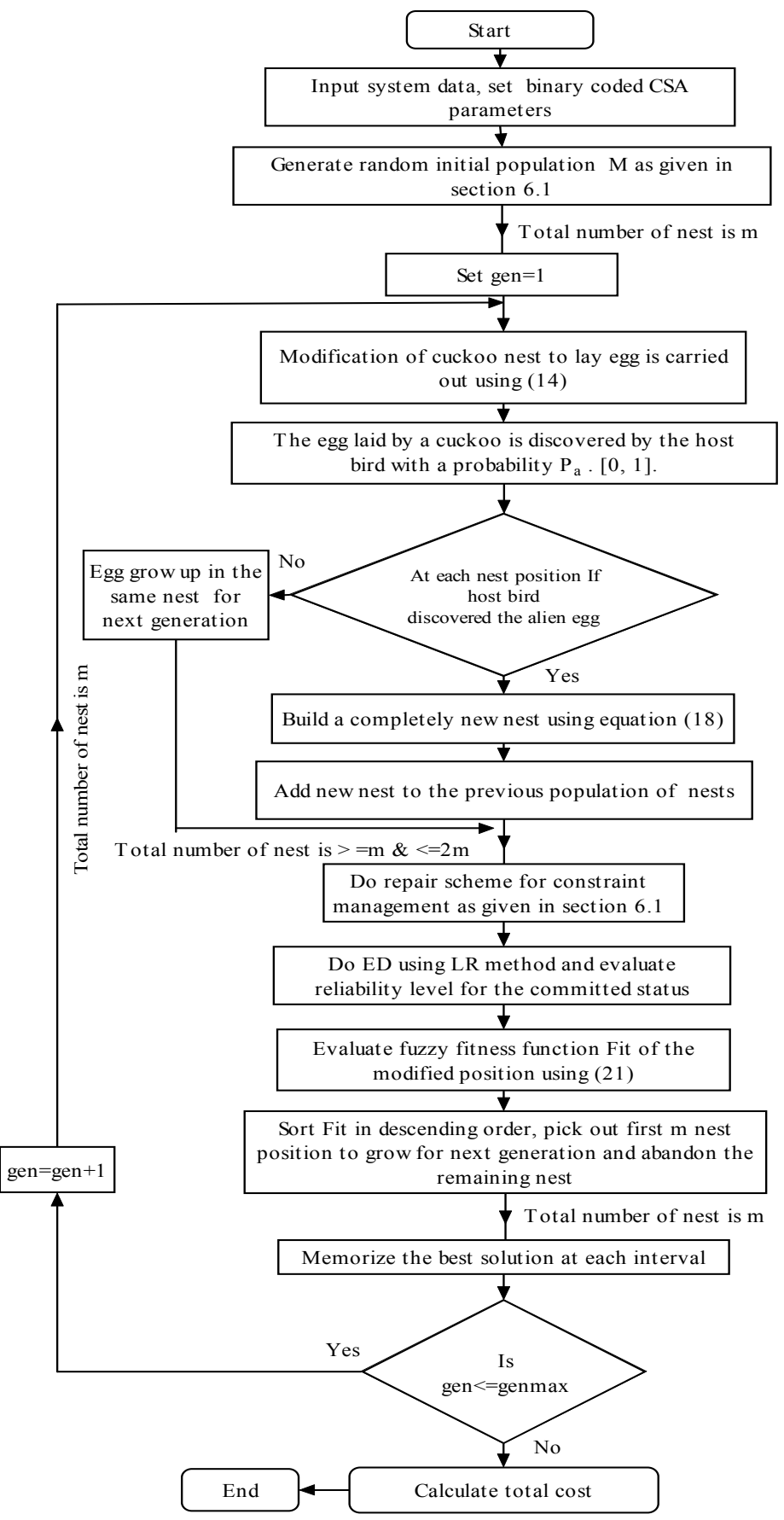

\section{Results and Discussions}

Fig. 4 Flowchart for MOUCP using fuzzy integrated CSA

All the programs are developed using MATLAB 7.01.The system configuration is Pentium IV processor with 3.2GHz speed and 1GB RAM. Three case studies are considered to demonstrate the proposed methodology. In case 1, to validate the binary CSA, UCP is solved and the results are compared with existing literature. In case 2, to demonstrate the necessity of MOUCP, reliability constrained UCP is solved and the results are compared with existing literature. In case 3, to validate fuzzy integrated binary CSA, two objective functions (i.e. fuel cost and reliability function) for MOUCP are considered.

7.1 Case 1

In this case, unit commitment problem is solved as single objective with cost minimization problem. The applicability and validity of the CSA for practical applications have been tested on IEEE RTS 26- thermal power unit system (Wong et. al., 1999; Wang and Shaidehpour, 1993). The generation cost coefficient and the load profile is adapted from the (Wang and Shaidehpour, 1993). To validate the efficiency in exploring the global or near-global optima the power balance, power generation limits, and ramp rate limit constraints alone are included for the sake of comparison with other techniques reported in literature. To validate 
the computational efficiency of the proposed CSA method, the same problem is solved using simple real coded GA (Chandrasekaran, 2010) and standard PSO algorithm (Chandrasekaran, 2009). The optimal parameters obtained by trial and error for PSO and GA are as follows:

\section{PSO parameters}

Population size $=100$

Acceleration coefficients,

$\mathrm{c} 1=0.2, \mathrm{c} 2=0.2$

Inertia weight: $\mathrm{Wmax}=0.9, \mathrm{Wmin}=0.2$

\section{GA parameters}

Population size $=100$

Mutation rate $=0.01$

Crossover rate $=0.7$

The test system given in ref. (Wang et. al., 1999; Wang. and Shaidehpour, 1993) is considered and the system spinning reserve is deterministically set to the maximum capacity of the largest committed thermal power unit (i.e $400 \mathrm{MW}$ ) as in (Wang. and Shaidehpour (1993); Ongsakul and Petcharaks, 2004; Dieu and Ongsakul, 2008). Here UC problem is solved using the proposed binary coded CSA and Lagrangian multiplier method is used to solve the economic dispatch problem.

Table 1 Comparison of results - Case 1

\begin{tabular}{lccc}
\hline Solution Techniques & Minimum value (\$) & Mean value (\$) & Maximum value (\$) \\
\hline ANN-DP (Wang, and Shaidehpour, 1993) & 729326.50 & - & - \\
ILR (Ongsakul W and Petcharaks N. 2004) & 725996.90 & - & - \\
IPL-ALH (Dieu and Ongsakul., 2008) & 721352.90 & - & - \\
GA & 721322.53 & 721635.14 & 721796.21 \\
PSO & 721214.32 & 721482.33 & 721666.21 \\
Binary CSA & 721186.20 & 721286.69 & 721577.39 \\
\hline
\end{tabular}

Table 2 Generation schedule - Case 1

\begin{tabular}{|c|c|c|c|c|c|c|c|c|c|c|c|c|c|c|c|c|c|c|c|c|c|c|c|c|c|c|c|}
\hline Unit no. & \multicolumn{26}{|c|}{ Thermal power unit status } & Operating cost (\$) \\
\hline 1 & 1 & 1 & 1 & 1 & 1 & 1 & 1 & 1 & 1 & 1 & 1 & 1 & 0 & 0 & 0 & 0 & 0 & 0 & 0 & 0 & 0 & 0 & 0 & 0 & 0 & 0 & 19121.90 \\
\hline 2 & 1 & 1 & 1 & 1 & 1 & 1 & 1 & 1 & 1 & 1 & 1 & 1 & 0 & 0 & 0 & 0 & 0 & 0 & 0 & 0 & 0 & 0 & 0 & 0 & 0 & 0 & 19371.00 \\
\hline 3 & 1 & 1 & 1 & 1 & 1 & 1 & 1 & 1 & 1 & 1 & 0 & 1 & 0 & 0 & 0 & 0 & 0 & 0 & 0 & 0 & 0 & 0 & 0 & 0 & 0 & 0 & 18789.00 \\
\hline 4 & 1 & 1 & 1 & 1 & 1 & 1 & 1 & 1 & 1 & 1 & 0 & 1 & 0 & 0 & 0 & 0 & 0 & 1 & 0 & 0 & 0 & 0 & 0 & 0 & 0 & 0 & 18966.00 \\
\hline 5 & 1 & 1 & 1 & 1 & 1 & 1 & 1 & 1 & 1 & 1 & 1 & 1 & 0 & 0 & 0 & 0 & 0 & 0 & 0 & 0 & 0 & 0 & 0 & 0 & 0 & 0 & 19684.33 \\
\hline 6 & 1 & 1 & 1 & 1 & 1 & 1 & 1 & 1 & 1 & 1 & 1 & 1 & 1 & 0 & 0 & 0 & 0 & 0 & 0 & 0 & 0 & 0 & 0 & 0 & 0 & 0 & 21317.50 \\
\hline 7 & 1 & 1 & 1 & 1 & 1 & 1 & 1 & 1 & 1 & 1 & 1 & 1 & 1 & 1 & 0 & 0 & 0 & 1 & 1 & 1 & 0 & 0 & 0 & 0 & 0 & 0 & 23830.60 \\
\hline 8 & 1 & 1 & 1 & 1 & 1 & 1 & 1 & 1 & 1 & 1 & 1 & 1 & 1 & 1 & 1 & 1 & 1 & 0 & 0 & 0 & 0 & 0 & 0 & 0 & 0 & 0 & 33691.00 \\
\hline 9 & 1 & 1 & 1 & 1 & 1 & 1 & 1 & 1 & 1 & 1 & 1 & 1 & 1 & 1 & 1 & 1 & 1 & 0 & 0 & 0 & 0 & 0 & 0 & 0 & 0 & 0 & 34726.00 \\
\hline 10 & 1 & 1 & 1 & 1 & 1 & 1 & 1 & 1 & 1 & 1 & 1 & 1 & 1 & 1 & 1 & 1 & 1 & 1 & 1 & 1 & 0 & 0 & 0 & 0 & 0 & 0 & 36050.00 \\
\hline 11 & 1 & 1 & 1 & 1 & 1 & 1 & 1 & 1 & 1 & 1 & 1 & 1 & 1 & 1 & 1 & 1 & 1 & 1 & 1 & 1 & 1 & 0 & 1 & 1 & 1 & 0 & 38370.80 \\
\hline 12 & 1 & 1 & 1 & 1 & 1 & 1 & 1 & 1 & 1 & 1 & 1 & 1 & 1 & 1 & 1 & 1 & 1 & 1 & 1 & 1 & 0 & 0 & 0 & 0 & 0 & 0 & 35816.00 \\
\hline 13 & 1 & 1 & 1 & 1 & 1 & 1 & 1 & 1 & 1 & 1 & 1 & 1 & 1 & 1 & 1 & 1 & 1 & 1 & 1 & 1 & 0 & 0 & 0 & 0 & 0 & 0 & 35816.00 \\
\hline 14 & 1 & 1 & 1 & 1 & 1 & 1 & 1 & 1 & 1 & 1 & 1 & 1 & 1 & 1 & 1 & 1 & 1 & 0 & 0 & 0 & 0 & 0 & 0 & 0 & 0 & 0 & 34917.00 \\
\hline 15 & 1 & 1 & 1 & 1 & 1 & 1 & 1 & 1 & 1 & 1 & 1 & 1 & 1 & 1 & 1 & 1 & 1 & 1 & 1 & 1 & 1 & 1 & 0 & 0 & 0 & 0 & 36582.00 \\
\hline 16 & 1 & 1 & 1 & 1 & 1 & 1 & 1 & 1 & 1 & 1 & 1 & 1 & 1 & 1 & 1 & 1 & 1 & 1 & 1 & 1 & 1 & 0 & 0 & 1 & 1 & 0 & 37680.59 \\
\hline 17 & 1 & 1 & 1 & 1 & 1 & 1 & 1 & 1 & 1 & 1 & 1 & 1 & 1 & 1 & 1 & 1 & 1 & 0 & 0 & 0 & 0 & 0 & 0 & 0 & 0 & 0 & 34917.00 \\
\hline 18 & 1 & 1 & 1 & 1 & 1 & 1 & 1 & 1 & 1 & 1 & 1 & 1 & 1 & 1 & 1 & 1 & 1 & 0 & 0 & 0 & 0 & 0 & 0 & 0 & 0 & 0 & 34534.00 \\
\hline 19 & 1 & 1 & 1 & 1 & 1 & 1 & 1 & 1 & 1 & 1 & 1 & 1 & 1 & 1 & 1 & 1 & 1 & 0 & 0 & 0 & 0 & 0 & 0 & 0 & 0 & 0 & 33963.00 \\
\hline 20 & 1 & 1 & 1 & 1 & 1 & 1 & 1 & 1 & 1 & 1 & 1 & 1 & 1 & 1 & 1 & 1 & 1 & 0 & 0 & 0 & 0 & 0 & 0 & 0 & 0 & 0 & 34917.00 \\
\hline 21 & 1 & 1 & 1 & 1 & 1 & 1 & 1 & 1 & 1 & 1 & 1 & 1 & 1 & 1 & 1 & 1 & 1 & 1 & 1 & 1 & 0 & 0 & 0 & 0 & 0 & 0 & 36050.00 \\
\hline 22 & 1 & 1 & 1 & 1 & 1 & 1 & 1 & 1 & 1 & 1 & 1 & 1 & 1 & 1 & 1 & 1 & 1 & 0 & 0 & 0 & 0 & 0 & 0 & 0 & 0 & 0 & 33584.00 \\
\hline 23 & 1 & 1 & 1 & 1 & 1 & 1 & 1 & 1 & 1 & 1 & 1 & 1 & 1 & 1 & 1 & 0 & 0 & 1 & 0 & 0 & 1 & 1 & 0 & 0 & 0 & 0 & 27426.00 \\
\hline 24 & 1 & 1 & 1 & 1 & 1 & 1 & 1 & 1 & 1 & 1 & 1 & 1 & 1 & 0 & 0 & 0 & 0 & 0 & 0 & 0 & 0 & 0 & 0 & 0 & 0 & 0 & 21066.00 \\
\hline & & & & & & & & & & & & & & 8 & & & & & & & & & & & & & 721186.20 \\
\hline
\end{tabular}


Table 1 gives the comparison of the minimum total operating cost obtained by CSA with respect to other techniques reported in the literature. It is clearly seen that the proposed method yields better results than other techniques, so far proposed in the literature. The minimum cost so far reported in literature is $\$ 721352.9$ (Vo Ngoc Dieu and Weerakorn Ongsakul., 2008) which is $\$ 166.7$ higher than that obtained from cuckoo search. The details of the power dispatch for case 1 is given in Table 2 .

\subsubsection{Solution Quality and Computation efficiency}

Fig. 5 shows the convergence graph of minimum operating cost obtained from 30 trial runs for case 1 using GA, PSO, and CSA. It is inferred from Fig. 5 that the characteristics of binary coded CSA algorithm steadily reaches the minimum value after few iterations when compared to other two methods (GA and PSO). This shows that the proposed CSA method has better quality of solution. However, in PSO and GA the characteristics exhibit premature convergence and settles to near-global optima. In all the three methods, the maximum number of iteration is fixed as 300 .

To compare the computation time of the proposed algorithm, the UCP is solved using GA and PSO. Although real coded GA has better computation time than PSO, GA struck to local optima that degrades its performance. In all the three methods, the maximum number of iteration is fixed as 300 generation and population size as 100 . Comparison of computation time for the three cases is shown in Table 3. It can be seen that the time taken by the CSA is lesser than that of GA and PSO.

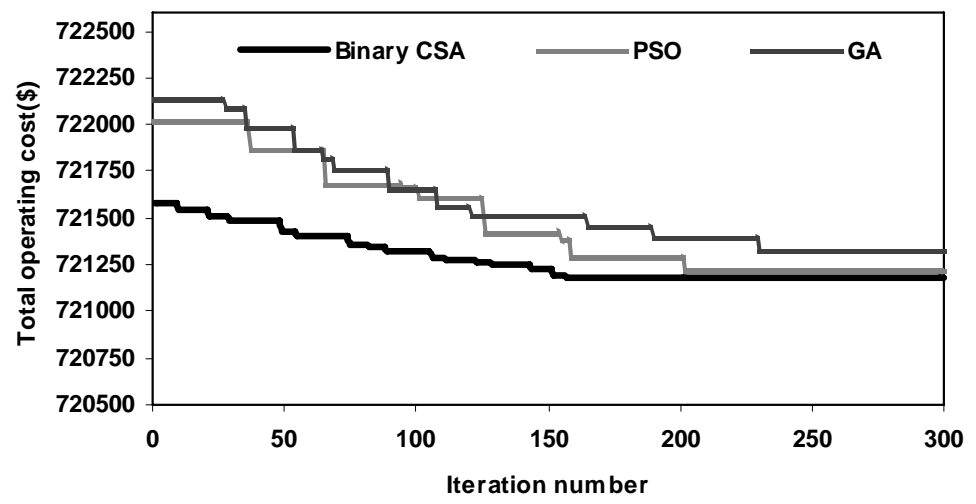

Fig.5 Convergence graph for 26 thermal power unit system- Case 1

\subsubsection{Effect of Variation of CSA Parameters.}

Good converge behavior can be obtained if the three control parameters namely cuckoo nest population size, maximum generation and $\lambda$, can be optimally tuned. Setting of these cuckoo parameters optimally would also yield better solution and lesser computational time. In order to avoid misleading results due to the breeding behavior of cuckoos of the CSA, several test runs are carried out to set the cuckoo nest population size. 10 trial runs are carried out for the test case 1 . The nest population size is then varied between 25 and 300 for the maximum generation of 500 and $\lambda$ is set as 1 .

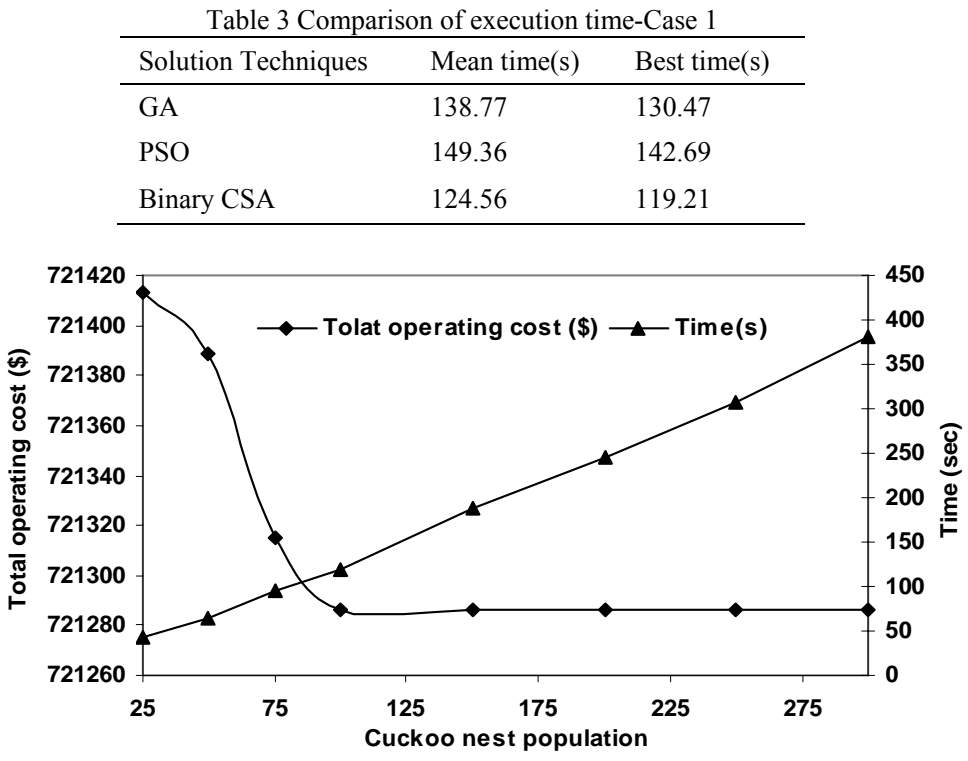

Fig. 6 Average of total operating cost for different cuckoo nest sizes-Case1 
Fig. 6 shows the average value of total operating cost out of 10 trials for different values of nest size. With increase in nest size, a steady improvement in average cost is noticed. When the nest size is greater than 100 , there is no significant improvement in the average of total operating cost. Also, in general when there is an increase in the nest size, the number of generation required by the CSA to converge to the optimum solution decreases. On the other hand, the CPU time required for the evaluation of generation increases almost linearly with the nest size which is shown in Fig. 6. Thereby, an intermediate value of 100 is chosen for the nest size, which gives an increase in efficiency and helps in finding global best solution. Thus, the best possible setting for the population size is chosen as 100 .

Also for the population size 100 and $\lambda=1$, the maximum generation is varied from 50 to 400 . From the Fig. 7, it is observed with increase in maximum generation limit a steady improvement in average cost is noticed. When the maximum generation is greater than 250 , there is no significant improvement in the total operating cost. Thus, the best possible setting for the maximum number of generation is set as 300 for the nest population size 100 .

Finally $\lambda$ is varied for the nest population size of 100 and maximum generation is set as 300 . The Table 4 gives the average cost values obtained in ten trails for the different values of $\lambda$. From the Table 4 it is concluded the best $\lambda$ value chosen is 1 for case 1 . Hence the nest population size as 100 , maximum generation limit as 300 and $\lambda=1$ is chosen as best parameter for this system.

\subsection{Case 2}

In case 2, for the same IEEE RTS 26 thermal power unit system, the system spinning reserve is calculated by predefining the system reliability level as a constraint and the reliability constrained UCP with cost minimization is solved using binary coded CSA. Here UCP is solved using the proposed binary coded CSA and Lagrangian multiplier is used to solve the EDP. Here the reliability level of the system is considered as constraint in the UCP. In (Simopoulos D, 2006), loss of load probability (LOLP) index is used to evaluate the reliability of the system. Also LOLP index is expressed in $\%$ and the lead time of the system is fixed for 4 hours.

Table 5 gives the comparison of the minimum total operating cost obtained by CSA with respect to other techniques reported in the literature for the $L O L P_{\text {spec }}=1 \%$ and $0.5 \%$. It can be inferred from the results that the solution obtained from the proposed method is better than the SA, GA and PSO algorithm for the different value of $L O L P_{\text {spec. }}$ CSA finds a solution which is $\$ 147$ and $\$ 474$ lesser when $L O L P_{\text {spec }}$ is $1 \%$ and $0.5 \%$ respectively, and is found to be promising. The details of the power dispatch for each thermal power unit of the 24 bus system for $L O L P_{\text {spec }}=0.5 \%$ is given in Table 6 .

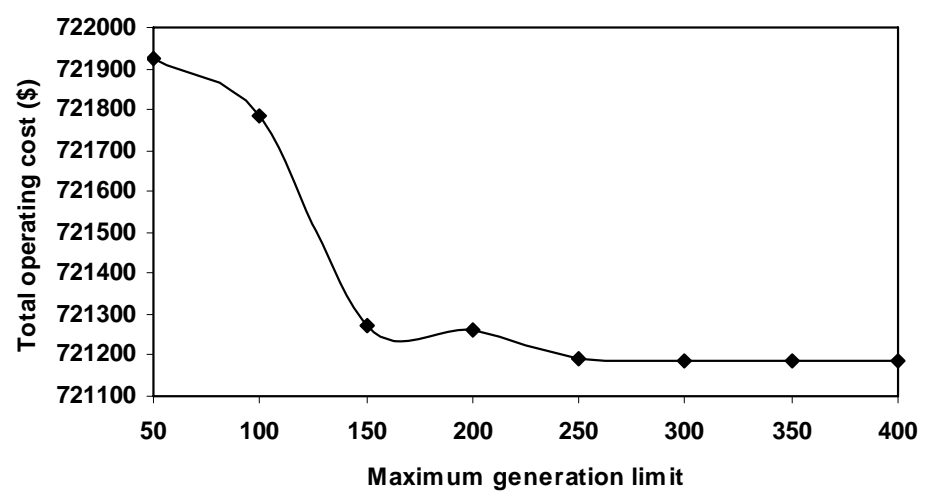

Fig. 7 Variation of average operating cost with maximum generation-Case 1

\begin{tabular}{|c|c|}
\hline$\lambda$ & $\begin{array}{l}\text { Average } \\
\text { Cost }(\$)\end{array}$ \\
\hline 0.5 & 721197.3 \\
\hline 1 & 721196.2 \\
\hline 1.5 & 721211.9 \\
\hline 2 & 721210.1 \\
\hline 2.5 & 721197.3 \\
\hline 3 & 721206.6 \\
\hline
\end{tabular}


Table 5 Comparison of results - Case 2

\begin{tabular}{ccccccc}
\hline \multirow{2}{*}{ Solution techniques } & \multicolumn{3}{c}{$L O L P=1 \%$} & \multicolumn{3}{c}{$L O L P=0.5 \%$} \\
\cline { 2 - 7 } & $\begin{array}{c}\text { Minimum } \\
\text { value }\end{array}$ & $\begin{array}{c}\text { Mean } \\
\text { value }\end{array}$ & $\begin{array}{c}\text { Maximum } \\
\text { value }\end{array}$ & $\begin{array}{c}\text { Minimum } \\
\text { value }\end{array}$ & $\begin{array}{c}\text { Mean } \\
\text { value }\end{array}$ & $\begin{array}{c}\text { Maximum } \\
\text { value }\end{array}$ \\
\hline SA(Simopoulos D, 2006) & 717938 & 718039 & 718135 & 722401 & 722622 & 723180 \\
GA & 717967 & 718643 & 719035 & 722466 & 722790 & 723274 \\
PSO & 717826 & 718061 & 718667 & 722358 & 722715 & 723154 \\
Binary CSA & 717791 & 718143 & 718921 & 721927 & 722126 & 722497 \\
\hline
\end{tabular}

It is observed from Table 5, that the cost of operation is well correlated with predefined reliability level i.e the operating cost is found to increase as the $L O L P$ criterion $\left(L O L P_{\text {spec }}\right)$ is restricted. Also when the $L O L P_{\text {spec }}$ is $0.05 \%$, the UCP solution (cost) is $\$ 724391$ and further decrement of $L O L P_{\text {spec }}$ has no effect on the operating cost. Since this $L O L P_{\text {spec }}$ is the maximum reliability level supplied by the generation system. Similarly, $L O L P_{\text {spec }}=5.5 \%$ is the minimum reliability level supplied by the generation system. However, increase or decrease in the reliability level depends on the choice of defining $L O L P_{\text {spec }}$ by the system operator. Here low value of $L O L P_{\text {spec }}$ means a system maintaining high reliability and vice versa. Therefore the system operator will set the reliability level based on the trade of between the cost and reliability level.

If defined the reliability index of the system should be between $4 \%$ (minimum) and $1 \%$ (maximum) in the entire scheduling period. In this case, any algorithm will fail to give the solution, since in the $9^{\text {th }}$ hour (for the load $2540 \mathrm{MW}$ ) it is not possible to bring the reliability level within the specified range. This is because when committing additional thermal power unit to increase reliability level (i.e to reduce the $L O L P$ index), the evaluated reliability level is less than $1 \%$ and violate the minimum limit. Also for all other combination of committing the thermal power unit, it is unable to bring the reliability level within the minimum and maximum range. Similarly for any other limit of reliability constraints, the system failed to satisfy the reliability level within the range in any one or more than one hour.

From the case 1 and case 2, it is clearly observed that the above nature setting of spinning reserve by deterministic and probabilistic method does not give a satisfactory UCP solution. Therefore it stresses the need for the formulation of MOUCP to obtain a best compromise solution between the cost and the reliability level.

Table 6 Generation schedule for reliability level $L O L P_{\text {spec }}=0.5 \%$ - Case 2

\begin{tabular}{|c|c|c|c|c|c|c|c|c|c|c|c|c|c|c|c|c|c|c|c|c|c|c|c|c|c|c|c|}
\hline Unit no. & \multicolumn{26}{|c|}{ Thermal power unit status } & Operating cost (\$) \\
\hline 1 & 1 & 1 & 1 & 1 & 1 & 1 & 1 & 1 & 1 & 1 & 1 & 1 & 0 & 0 & 0 & 0 & 0 & 0 & 0 & 0 & 0 & 0 & 0 & 0 & 0 & 0 & 19121.90 \\
\hline 2 & 1 & 1 & 1 & 1 & 1 & 1 & 1 & 1 & 1 & 1 & 1 & 1 & 0 & 0 & 0 & 0 & 0 & 0 & 0 & 0 & 0 & 0 & 0 & 0 & 0 & 0 & 19371.00 \\
\hline 3 & 1 & 1 & 1 & 1 & 1 & 1 & 1 & 1 & 1 & 1 & 1 & 1 & 0 & 0 & 0 & 0 & 0 & 0 & 0 & 0 & 0 & 0 & 0 & 0 & 0 & 0 & 18896.00 \\
\hline 4 & 1 & 1 & 1 & 1 & 1 & 1 & 1 & 1 & 1 & 1 & 1 & 1 & 0 & 0 & 0 & 0 & 0 & 0 & 0 & 0 & 0 & 0 & 0 & 0 & 0 & 0 & 19015.00 \\
\hline 5 & 1 & 1 & 1 & 1 & 1 & 1 & 1 & 1 & 1 & 1 & 1 & 1 & 0 & 0 & 0 & 0 & 0 & 0 & 0 & 0 & 0 & 0 & 0 & 0 & 0 & 0 & 19610.00 \\
\hline 6 & 1 & 1 & 1 & 1 & 1 & 1 & 1 & 1 & 1 & 1 & 1 & 1 & 1 & 0 & 0 & 0 & 0 & 0 & 0 & 0 & 0 & 0 & 0 & 0 & 0 & 0 & 21317.50 \\
\hline 7 & 1 & 1 & 1 & 1 & 1 & 1 & 1 & 1 & 1 & 1 & 1 & 1 & 1 & 0 & 1 & 0 & 0 & 0 & 0 & 0 & 0 & 0 & 0 & 0 & 0 & 0 & 24451.70 \\
\hline 8 & 1 & 1 & 1 & 1 & 1 & 1 & 1 & 1 & 1 & 1 & 1 & 1 & 1 & 0 & 1 & 1 & 1 & 0 & 0 & 0 & 0 & 0 & 0 & 0 & 0 & 0 & 33139.90 \\
\hline 9 & 1 & 1 & 1 & 1 & 1 & 1 & 1 & 1 & 1 & 1 & 1 & 1 & 1 & 1 & 1 & 1 & 1 & 0 & 0 & 0 & 0 & 0 & 0 & 0 & 0 & 0 & 34861.50 \\
\hline 10 & 1 & 1 & 1 & 1 & 1 & 1 & 1 & 1 & 1 & 1 & 1 & 1 & 1 & 1 & 1 & 1 & 1 & 1 & 1 & 1 & 0 & 0 & 0 & 0 & 0 & 0 & 36050.00 \\
\hline 11 & 1 & 1 & 1 & 1 & 1 & 1 & 1 & 1 & 1 & 1 & 1 & 1 & 1 & 1 & 1 & 1 & 1 & 1 & 1 & 1 & 1 & 1 & 1 & 1 & 1 & 0 & 38402.80 \\
\hline 12 & 1 & 1 & 1 & 1 & 1 & 1 & 1 & 1 & 1 & 1 & 1 & 1 & 1 & 1 & 1 & 1 & 1 & 1 & 1 & 1 & 0 & 0 & 0 & 0 & 0 & 0 & 35816.00 \\
\hline 13 & 1 & 1 & 1 & 1 & 1 & 1 & 1 & 1 & 1 & 1 & 1 & 1 & 1 & 1 & 1 & 1 & 1 & 1 & 1 & 1 & 0 & 0 & 0 & 0 & 0 & 0 & 35816.00 \\
\hline 14 & 1 & 1 & 1 & 1 & 1 & 1 & 1 & 1 & 1 & 1 & 1 & 1 & 1 & 1 & 1 & 1 & 1 & 0 & 0 & 0 & 0 & 0 & 0 & 0 & 0 & 0 & 34917.00 \\
\hline 15 & 1 & 1 & 1 & 1 & 1 & 1 & 1 & 1 & 1 & 1 & 1 & 1 & 1 & 1 & 1 & 1 & 1 & 1 & 1 & 1 & 1 & 1 & 0 & 0 & 0 & 0 & 36582.00 \\
\hline 16 & 1 & 1 & 1 & 1 & 1 & 1 & 1 & 1 & 1 & 1 & 1 & 1 & 1 & 1 & 1 & 1 & 1 & 1 & 1 & 1 & 1 & 1 & 1 & 1 & 0 & 0 & 37710.59 \\
\hline 17 & 1 & 1 & 1 & 1 & 1 & 1 & 1 & 1 & 1 & 1 & 1 & 1 & 1 & 1 & 1 & 1 & 1 & 0 & 0 & 0 & 0 & 0 & 0 & 0 & 0 & 0 & 34917.00 \\
\hline 18 & 1 & 1 & 1 & 1 & 1 & 1 & 1 & 1 & 1 & 1 & 1 & 1 & 1 & 1 & 1 & 1 & 1 & 0 & 0 & 0 & 0 & 0 & 0 & 0 & 0 & 0 & 34534.00 \\
\hline 19 & 1 & 1 & 1 & 1 & 1 & 1 & 1 & 1 & 1 & 1 & 1 & 1 & 1 & 1 & 1 & 1 & 1 & 0 & 0 & 0 & 0 & 0 & 0 & 0 & 0 & 0 & 33963.00 \\
\hline 20 & 1 & 1 & 1 & 1 & 1 & 1 & 1 & 1 & 1 & 1 & 1 & 1 & 1 & 1 & 1 & 1 & 1 & 0 & 0 & 0 & 0 & 0 & 0 & 0 & 0 & 0 & 34917.00 \\
\hline 21 & 1 & 1 & 1 & 1 & 1 & 1 & 1 & 1 & 1 & 1 & 1 & 1 & 1 & 1 & 1 & 1 & 1 & 1 & 1 & 1 & 0 & 0 & 0 & 0 & 0 & 0 & 36050.00 \\
\hline 22 & 1 & 1 & 1 & 1 & 1 & 1 & 1 & 1 & 1 & 1 & 1 & 1 & 1 & 1 & 1 & 1 & 1 & 0 & 0 & 0 & 0 & 0 & 0 & 0 & 0 & 0 & 33584.00 \\
\hline 23 & 1 & 1 & 1 & 1 & 1 & 1 & 1 & 1 & 1 & 1 & 1 & 1 & 1 & 0 & 1 & 1 & 0 & 0 & 0 & 0 & 0 & 0 & 0 & 0 & 0 & 0 & 27819.00 \\
\hline 24 & 1 & 1 & 1 & 1 & 1 & 1 & 1 & 1 & 1 & 1 & 1 & 1 & 1 & 0 & 0 & 0 & 0 & 0 & 0 & 0 & 0 & 0 & 0 & 0 & 0 & 0 & 21066.00 \\
\hline & & & & & & & & & & & & & & & & & & & & & & 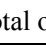 & & & & & 721928.90 \\
\hline
\end{tabular}




\subsection{Case 3}

In case 3, for the same IEEE RTS 26 thermal power unit system, a proposed MOUCP is solved using fuzzy integrated BCSL. As there are 2 objective functions, three possible combinations of objection function are obtained. The UCP solution for case 3 is given in Table 7. When UCP is solely solved for the minimization of $F_{c}$, minimum cost of $\$ 704160$ is obtained by neglecting other objectives. When fuel cost is alone considered, a downward trend is seen in the reliability level of the system. Since the thermal power units are committed solely based on the cost, the reliability of the system is very low due to the shortage of spinning reserve availability for the committed thermal power units.

When reliability is alone minimized, neglecting other objectives, the resultant reliability index LOLP is 0 . The maximum reliability level of the system is obtained with a higher fuel cost. This is because, commission of more number of thermal power units will increase the fuel cost, proportionally.

The above nature of conflicting objectives does not give a satisfactory UCP solution. Therefore it stresses the need for the formulation of MOUCP to obtain a best compromise solution.

Table 7 MOUCP solution using BCSL- Case 3

\begin{tabular}{cccc}
\hline Objective function & Minimization of $\mathrm{F}_{\mathrm{c}}$ & Minimization of LOLP & Compromise solution \\
\hline $\begin{array}{c}\text { Operating cost (\$) } \\
\text { LOLP maintained } \\
\text { at each hour }\end{array}$ & 704160 & 724391 & $\begin{array}{c}711981.27 \\
20 \%-50 \%\end{array}$ \\
& $5.5 \%$ & $0.05 \%$ & $\begin{array}{c}\text { of the available reliability } \\
\text { level at each hour. }\end{array}$ \\
\hline
\end{tabular}

Table 8 MOUCP solution using BCSL- Case 3

\begin{tabular}{|c|c|c|c|c|c|c|}
\hline Hour & Unit Status $1 \ldots .26$ & $\begin{array}{l}\text { Operating } \\
\text { cost } \$\end{array}$ & $\begin{array}{l}\text { Membership } \\
\text { value for } \\
\text { cost }\end{array}$ & $\begin{array}{l}\text { Maintained } \\
\text { LOLP }\end{array}$ & $\begin{array}{l}\text { Membership } \\
\text { value for } \\
L O L P\end{array}$ & $\begin{array}{c}\text { Committed } \\
\text { Spinning } \\
\text { reserve, } \\
\text { MW }\end{array}$ \\
\hline 1 & 11111111100000000000000000 & 18429.00 & 1 & 0.0108641 & 0.0622 & 222 \\
\hline 2 & 11111111100000000000000000 & 18789.00 & 1 & 0.0108641 & 0.0622 & 192 \\
\hline 3 & 11111111100000000000000000 & 18310.00 & 1 & 0.0107979 & 0.0374 & 232 \\
\hline 4 & 11111111110000000000000000 & 18615.60 & 1 & 0.0107978 & 0.0374 & 298 \\
\hline 5 & 11111111111000000000000000 & 19323.82 & 0.5948 & 0.0106966 & 1 & 324 \\
\hline 6 & 11111111111100000000000000 & 20947.53 & 0.4658 & 0.0106961 & 1 & 324 \\
\hline 7 & 11111111111110000000000000 & 23346.62 & 1 & 0.0107972 & 1 & 274 \\
\hline 8 & 11111111111111110000000000 & 32904.12 & 0.5325 & 0.0108531 & 1 & 338 \\
\hline 9 & 11111111111111110000000000 & 34298.00 & 1 & 0.0113877 & 0.3987 & 228 \\
\hline 10 & 11111111111111111000000000 & 36320.62 & 0.0125 & 0.0074675 & 0.101 & 365 \\
\hline 11 & 11111111111111111000000000 & 37608.00 & 1 & 0.0111764 & 0.5671 & 295 \\
\hline 12 & 11111111111111111000000000 & 35726.00 & 0.6798 & 0.0074666 & 0.1004 & 375 \\
\hline 13 & 11111111111111111000000000 & 35726.00 & 0.6798 & 0.0074666 & 0.1004 & 375 \\
\hline 14 & 11111111111111111000000000 & 34917.00 & 0 & 0.0005373 & 0 & 415 \\
\hline 15 & 11111111111111111000000000 & 36430.00 & 1 & 0.0109564 & 1 & 345 \\
\hline 16 & 11111111111111111000000000 & 37136.00 & 1 & 0.0109577 & 1 & 315 \\
\hline 17 & 11111111111111110000000000 & 34533.00 & 1 & 0.0113877 & 0.3987 & 218 \\
\hline 18 & 11111111111111110000000000 & 34063.00 & 1 & 0.0112584 & 0.3714 & 238 \\
\hline 19 & 11111111111111110000000000 & 33410.00 & 1 & 0.0110996 & 0.338 & 268 \\
\hline 20 & 11111111111111110000000000 & 34533.00 & 1 & 0.0113877 & 0.3987 & 218 \\
\hline 21 & 11111111111111110000000000 & 35712.00 & 1 & 0.0194979 & 0 & 168 \\
\hline 22 & 11111111111110110000000000 & 33141.00 & 0.4296 & 0.0193444 & 0.8723 & 188 \\
\hline 23 & 11111111110110100000000000 & 27248.00 & 0.6504 & 0.0151701 & 1 & 195 \\
\hline 24 & 11111111100100000000000000 & 20514.00 & 0.0405 & 0.0109168 & 1 & 182 \\
\hline
\end{tabular}

In the section a novel method of scheduling the spinning reserve is proposed, based on the available generation to satisfy the reliability level in each hour of the dispatch period. Based on the availability of the generation system, the fuzzy system made the decision to commit the spinning reserve at each hour. Since the load of system is varied at each hour, correspondingly the maximum reliability level of the system will be varied. Therefore with the assist of fuzzy system, the reliability level of the system will be maintained between some percentages of the available reliability level at each of the hours with respect to the cost. 
Here UCP is solved using the proposed binary coded CSA and Lagrangian multiplier is used to solve the EDP. The initial population of nest and maximum generation for binary coded CSA is set as 100 and 300, respectively. Out of 30 trails the best compromise solution of MOUCP solution is given in Table 7. The detailed dispatch for 24 hour time horizon is given in Table 8 .

From the thermal power unit status it is clearly understood that fuzzy set theory intelligently made the decision to schedule the compromise solution between the operating cost and the reliability level of the system. It is to be noted, in the $14^{\text {th }}$ hour the calculated reliability level is less than the $20 \%$ (maximum reliability) of $L O L P_{\min }$. Hence the membership value of $L O L P$ at $14^{\text {th }}$ hour is 0 . Increased reliability level will increase the operating cost thereby the corresponding membership value of cost is zero. Similarly in the $21^{\text {st }}$ hour, the reliability level is greater than the $50 \%$ (minimum reliability level) of $L O L P_{\min }$. Here the reliability level of that hour is compromised in supplying less operating cost, hence the cost is well within the design variable and membership value is set as 1 . This intelligent decision of MOUCP to schedule spinning reserve and to minimize the operating cost will eliminate the trade off between the cost and reliability level of the system.

The Fitness convergence graph for the case 3 is given in Fig. 8. From the figures, it is clear that the binary coded CSA is efficient for finding thermal power unit scheduling such that intelligent decision is made by the fuzzy set theory for the efficient trade off between the cost and reliability level of the system.

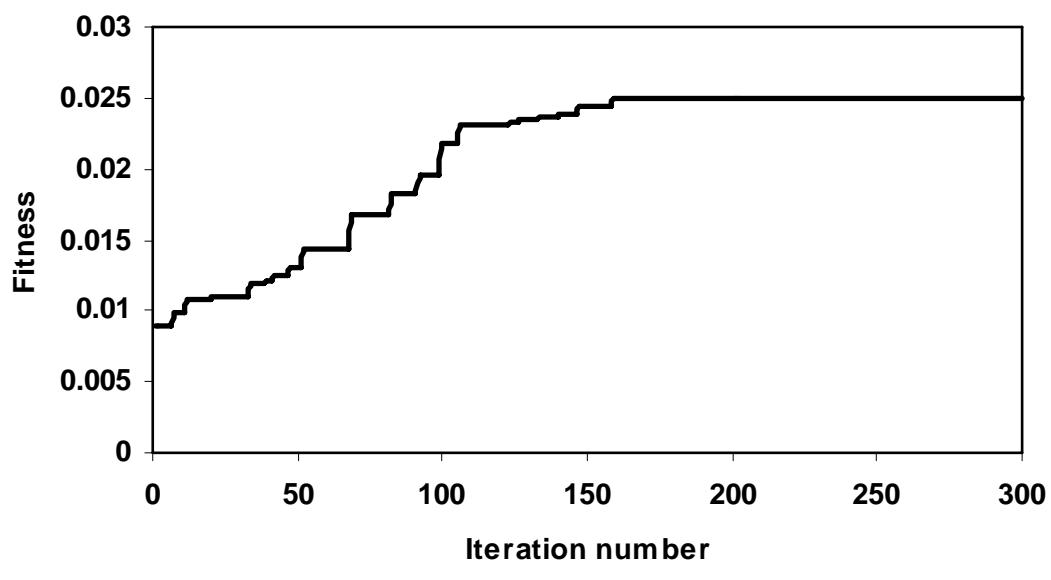

Fig. 8 Fitness convergence graph-Case 3

\section{Conclusion}

This paper has employed the special characteristic of cuckoos in searching the nest to lay eggs is developed and motivated for solving the single and multi-objective unit commitment problem.

- The BCSL algorithm when applied to practical UCP outperforms with the other techniques reported in the literature in obtaining minimum cost solution in less computational time.

- The significance of reliability level of the system is studied, and the novel MOUCP is formulated.

- Finally, the proposed fuzzy integrated BCSL algorithm has been presented for solving MOUCP. The problem has been formulated as a MOOP with competing fuel cost and reliability objective functions. The limitation (i.e. trade of between the cost and reliability level of the system) in selecting a feasible solution from a set of population solutions are circumvented by integrating fuzzy set theory and Binary CSA (heuristic approach).

The comparison of the results with other methods reported in the literature shows the superiority of the proposed methodology and its potential for solving conflicting objective function in a power system. The method is promising, simple, easy to implement and applicable for any large-scale power systems.

\section{Nomenclature}

$F_{c} \quad$ Fuel cost function (\$).

$a(i), b(i), c(i) \quad$ Cost coefficient of $i^{\text {th }}$ thermal power unit.

$H \quad$ Total number of hours considered.

$I(i, k) \quad$ Status of thermal power unit $i$ at $k^{\text {th }}$ hour. (i.e.) 1 for ON and 0 for OFF.

$L O L P(k) \quad$ Reliability function (loss of load probability).

Load( $k) \quad$ Total system demand at $k^{\text {th }}$ hour.

$N \quad$ Total number of thermal power units.

$P(i, k) \quad$ Generation power output of thermal power unit $i$ at $k^{\text {th }}$ hour.

$p(j) \quad$ Probability for state $i$. 


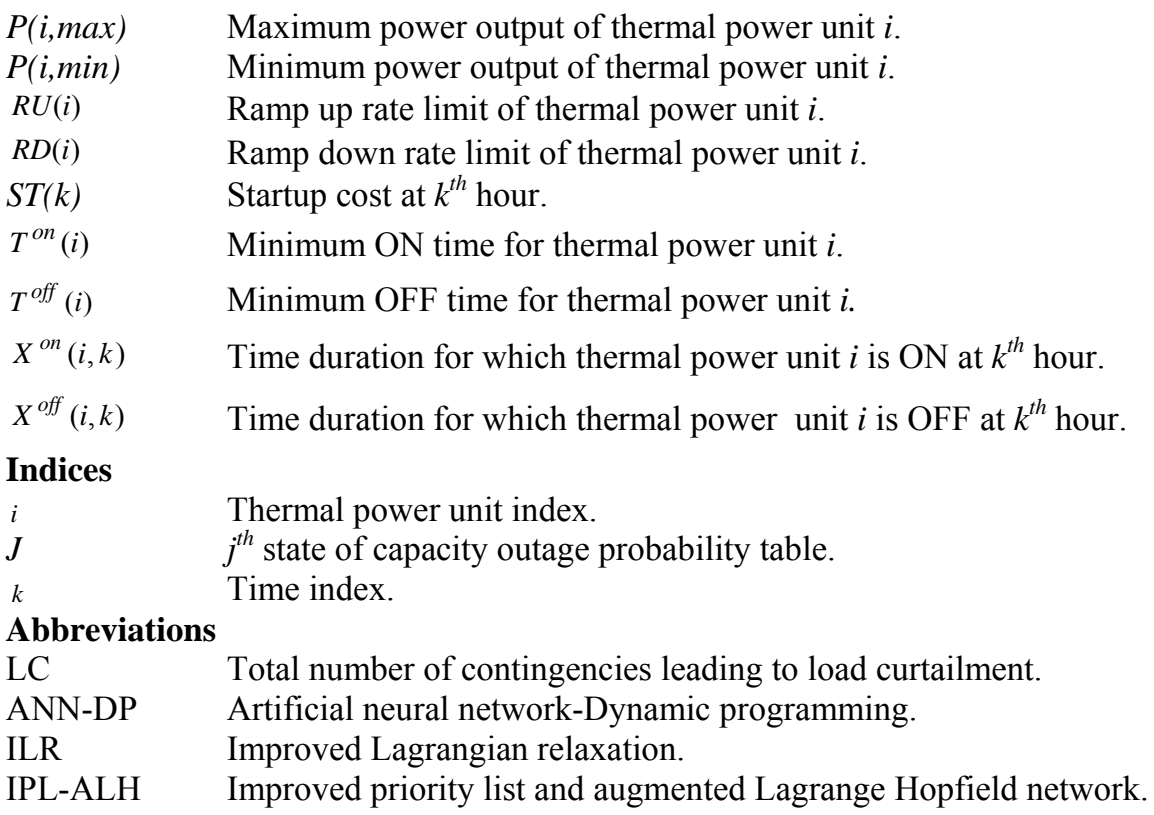

\section{References}

Bouffard F. and Galiana F. D., 2004. An electricity market with a probabilistic spinning reserve criterion. IEEE Trans. Power Syst., Vol. 19, pp. 300-307.

Burns R. M. and Gibson C. A., 1975. Optimization of priority lists for a unit commitment program. In: Proceedings of IEEE PES (summer meeting), Vol. 75, pp. 453-461.

Cai J.J., Ma XQ, Li Q, Li LX and Peng HP., 2010. A multi-objective chaotic ant swarm optimization for environment/economic dispatch. Electrical Power and Energy System, Vol. 32, pp. 337-344.

Chandrasekaran K. and Simon S.P., 2010. Reliability constrained unit commitment using genetic algorithm. Recent Advancement in Electrical Science (ICRAES'10), pp. 115-26.

Chandrasekaran K., Jeyaraj K.A., Sahayasenthamil L. and Saravanan M., 2009. A new method to incorporate facts devices in optimal power flow using particle swarm optimization. Journal of Theoretical and Applied Information Technology, Vol. 5, No. 1, pp. 67-74.

Chattopadhyay C. and Baldick R., 2002. Unit commitment with probabilistic reserve. Proc. IEEE Power Eng. Soc. Winter Meeting, Vol. 1, pp. 280-285.

Chen C. L. and Wang S. C., 1993. Branch-and-bound scheduling for thermal generating units. IEEE Trans. Energy Convers, Vol. 8, pp. 184-189.

Cohen A.I. and Yoshimura M., 1983. A branch-and-bound algorithm for unit commitment. IEEE Trans. Power Apparatus Syst., Vol. 102, pp. 444-451.

Dasgupta D. and McGregor D.R., 1994. Thermal unit commitment using genetic algorithms. IEE Proc. Gener. Transm. Distrib., Vol. 141, pp. 459-465.

Dieu V.N. and Ongsakul W., 2008. Ramp rate constrained unit commitment by improved priority list and augmented Lagrange Hopfield network. Electric Power Systems Research, Vol. 78, pp. 291-301

Dillon T.S., Edwin, K.W., Kochs H.D., and Taud R. J., 1978. Integer programming approach to the problem of optimal unit commitment with probabilistic reserve determination. IEEE Trans. Power App. Syst., Vol. 97, pp. 2154-2166.

Gandomi A.H. and Alavi A.H., 2012. Krill herd: A new bio-inspired optimization algorithm, Commun Nonlinear Sci. Numer. Simulat., Vol. 17, pp. 4831-4845.

Gooi H. B., Mendes D. P., Bell K. R. W. and Kirschen D. S., 1999. Optimal scheduling of spinning reserve. IEEE Trans. Power Syst., Vol. 14, pp. 1485-1492.

Hemamalini S and Simon S.P., 2012. Emission constrained economic dispatch with 36 valve-point effect using Maclaurin series based Lagrangian method, International Journal of Power and Energy Conversion, Vol. 3, pp. 1-18.

Juste K.A., Kita H., Tanaka E. and Hasegawa J., 1999. An evolutionary programming to the unit commitment problem. IEEE Trans. Power Syst., Vol. 14, pp. 1452-1459.

Kazarlis S. A., Bakirtzis A.G., and Petridis V., 1996. A genetic algorithm solution to the unit commitment problem. IEEE Trans. Power Syst. Vol. 11, pp. 83-92. 
Lin W.-M., Gow H.-J., and Tsai M.-T., 2011. An efficient hybrid Taguchi-immune algorithm for the unit commitment problem. Expert Systems with Applications, Vol. 38, No. 11, pp. 13662-13669.

Mantaway, A.H., Abdel-Magid, Y.L. and Selim S.Z., 1998a. A simulated annealing algorithm for unit commitment. IEEE Trans. Power Syst., Vol. 13, pp. 197-204.

Mantaway A.H., Abdel-Magid, Y.L. and Selim S.Z., 1998b. Unit commitment by tabu search. IEE Proc Gener. Transm. Distrib., Vol. 145, pp. 56-64.

Muchkstadt J. A. and Wilson R. C., 1968. An application of mixed-integer programming duality to scheduling thermal generating systems. IEEE Trans. Power Syst., Vol. 87, pp. 1968-1978.

Ongsakul W and Petcharaks N., 2004. Ramp rate constrained unit commitment by improved adaptive Lagrangian relaxation. In: Proceedings of the Electricity Supply Industry in Transition: Issues and Prospect for Asia, Asian Institute of Technology.

Ouyang Z. and Shahidehpour M., 1992. A hybrid artificial neural network-dynamic programming approach to unit commitment. IEEE Trans. Power Syst., Vol. 7, pp. 236 - 242.

Padhy N.P., 004. Unit commitment-a bibliographical survey. IEEE Trans. Power Syst., Vol. 19, pp. 1196-1205.

Patra S. Goswami S.K. and Goswami B., 2009. Fuzzy and simulated annealing based dynamic programming for the unit commitment problem. Expert Systems with Applications Vol. 36, pp. 5081-5086.

Pavez-Lazo B.and Soto-Cartes J., 2011. A deterministic annular crossover genetic algorithm optimisation for the unit commitment problem. Expert Systems with Applications, Vol. 38, pp. 6523-6529.

Rajabioun R., 2011. Cuckoo optimization algorithm, Applied Soft Computing, Vol. 11, pp. 5508-5518.

Wong P., Albrecht P., Allan R., Bhavaraju M., Billinton R., Chen Q., Fong C., Haddad S., Kuruganty S., Li W., Mukerji R., Patton D., Rau N.,Reppen D., Schneider A., Shahidehpour M., Singh C., 1999. A report prepared by the reliability test system task force of the application of probability methods sub committee. IEEE Trans. Power Syst., Vol. 14, No. 3, pp. 1010-1020.

Saneifard S. Prasad N.R. and Smolleck H.A., 1997. A Fuzzy Logic Approach to Unit Commitment. IEEE Trans. Power Syst., vol. 12, pp. 988-995.

Sheble G.B. and Fahd, G.N., 1994. Unit commitment literature synopsis. IEEE Trans. Power Syst., Vol. 9, pp. 128-135.

Simon, S.P., Padhy N.P. and Anand, R.S., 2006. An ant colony system approach for unit commitment problem. Elect. Power Energy Syst. Vol. 28, pp. 315-323.

Simopoulos D. N., Kavatza S. D. and Vournas C. D.,2006. Reliability constrained unit commitment using simulated annealing. IEEE Trans. Power Syst., 21, 1699-1706.

Synder W. L., 1987. Dynamic programming approach to unit commitment. IEEE Trans. Power Syst. PWRS-2, pp. 339-350.

Saber A.Y., Senjyu T., Yona A. and Funabashi T., 2007. Unit commitment computation by fuzzy adaptive particle swarm optimization. IET Gener. Transm. Distrib. Vol. 1, pp. 456-465.

Ting T.O., Rao M.V.C. and Loo C.K., 2006. A novel approach for unit commitment problem via an effective hybrid particle swarm optimization. IEEE Trans. Power Syst., Vol. 21, No. 1, pp. 411-418.

Vinothkumar, K. and Selvan M. P., 2011. Fuzzy embedded genetic algorithm method for distributed generation planning. Electric Power Components and Systems, Vol. 39, pp. 346-366.

Wang C. and Shahidehpour M., 1992. A decomposition approach to non-linear multi-area generation scheduling with tie-line constraints using expert systems. IEEE Trans. Power Syst., vol. 7, pp. 1409-1418.

Wang, C. and Shaidehpour S. M., 1993. Effect of ramp-rate limits on unit commitment and economic dispatch. IEEE Trans. Power Syst., Vol. 8, pp. 1341-1350.

Wang, L.F. and Singh C., 2007. Environmental/economic power dispatch using a fuzzified multi-objective particle swarm optimization algorithm. Electr. Power Syst Res., Vol. 77, pp. 1654-1664.

Wang L. and Singh C., 2009. Unit commitment considering generator outages through a mixed-integer particle swarm optimization algorithm. Applied Soft Computing, Vol. 9, No. 3, pp. 947-953.

Wu L., Shaidehpour M. and Li T., 2008. Cost of reliability analysis based on stochastic unit commitment. IEEE Trans. Power Syst. Vol. 23, pp. 1364-1374.

Yuan X., Nie H., Su A., Wang L., and Yuan Y., 2009. An improved binary particle swarm optimization for unit commitment problem. Expert Systems with Applications, Vol. 36 pp. 8049-8055.

Yang, X.S. and Deb S., 2009. Cuckoo search via L'evy flights. Proc. of World Congress on Nature \& Biologically Inspired Computing (NaBIC 2009), IEEE Publications, USA, pp. 210-214.

Yang, X.S. and Deb S., 2010. Engineering optimization by cuckoo search. Int. J. Mathematical Modelling and Numerical Optimization, Vol. 1, pp. 330-343.

Zhuang F. and Galiana F.D., 1988. Towards a more rigorous and practical unit commitment by lagrangian relaxation. IEEE Trans. Power Syst., Vol. 3, pp. 763-773.

Zitzler, E. and Thiele L., 1999. Multiobjective evolutionary algorithms: a comparative case study and the strength pareto approach. IEEE Trans. Evol. Comput., Vol. 3, pp. 257-71. 
Bibliographies

K. Chandrasekaran, born in India, received his Bachelors of Engineering (Electrical and Electronics Engineering) and Masters of Engineering (Power system) at Anna University, Chennai, India in the year 2005 and 2007. He is currently pursuing his research work in the Department of Electrical and Electronics Engineering, National Institute of Technology, Tiruchirappalli, Tamil Nadu, India. His research interest includes Deregulation of Power system, Power Systems Planning and Reliability and AI.

Sishaj Pulikottil Simon, born in India, received his B.E and M.E in the year 1999 and 2001 respectively. He obtained his Ph.D., (Power System Engineering) at Indian Institute of Technology (IIT), Roorkee, India in the year 2006. Currently he is an Assistant professor in the Department of Electrical and Electronics Engineering at National Institute of Technology (NIT) (formerly Regional Engineering College), Tiruchirappalli, Tamil Nadu. His field of interest is in the area of Deregulation of Power System, Power System Operations and Control, Application of Artificial Intelligence, and New Optimization Techniques to Power System.

Received February 2012

Accepted August 2012

Final acceptance in revised form August 2012 